\section{Enhancement of the Dose on 12 MV Linac with Free Flattening Filter Mode}

\author{
Zeghari A. ${ }^{* \odot}$, Saaidi R. ${ }^{1}$, Cherkaoui El Moursli R. ${ }^{1}$
}

\begin{abstract}
Purpose: In the last years, some studies investigated dosimetric benefits of a free flattening filter for the photon mode in the radiotherapy field. This study aims to provide a theoretical study published and analysis of basic dosimetric properties for a Saturne 43 Linac head to implement free flattening filter beams clinically.

Material and Methods: This is the first Monte Carlo study for the head of Saturne 43 with replacement flattening filter mode investigating beam dosimetric characteristics, including central axis absorbed doses, beam profiles and photon energy spectra. The later ones were analyzed for flattening filter and replacement flattening filter beams using BEAMnrc and DOSXYZnrc Monte Carlo codes for $10 \times 10$ $\mathrm{cm}^{2}, 5 \times 5 \mathrm{~cm}^{2}$ and $2 \times 2 \mathrm{~cm}^{2}$ square field sizes.
\end{abstract}

Results: A 3.94-fold increase of dose rate and electron contaminating increased by $246.4 \%$ with the replacement flattening filter mode for field size of $10 \times 10 \mathrm{~cm}^{2}$. Reduction was made by replacement flattening filter beam in the peripheral dose up to $30 \%$, and the time was reduced more than $50 \%$.

Conclusion: Results obtained from our study revealed that some characteristic dosimetries such as the maximum increase in depth dose rate, decrease in out-ofdepth dose, and reducing time can be beneficial for the unflattened beam to be used in the radiotherapy for the advanced techniques.

Citation: Zeghari A, Saaidi R, Cherkaoui El Moursli R. Enhancement of the Dose on 12 MV Linac with Free Flattening Filter Mode. $J$ Biomed Phys Eng. 2019;9(4):437-444. https://doi.org/10.31661/jbpe.v0i0.924.

\section{Keywords}

Monte Carlo Method • Radiotherapy • Radiation Dosimetry • Stereotactic Radiation

\section{Introduction}

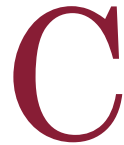

ancer has caused over $12 \%$ of all deaths in the world: more than 7 million people have died due to this disease annually. The latest estimate from the International Agency for Research on Cancer (IARC) has indicated that cancer incidence will reach a number of 22.2 million in 2030. Therefore, the cancer treatment will continue to be one of the greatest health challenges of the 21 st century. One of the most important and commonly used techniques is radiotherapy using the medical linear accelerators (Linac). In this linac, the angular distribution of the bremsstrahlung photons is modified by the component of flattening filter (FF). The latter is designed to generate a uniform lateral dose distribution inside a water phantom at the depth of $z=10 \mathrm{~cm}$. In the most clinical linac, the materials which formed the flattening filters are aluminium, Iron, copper, tungsten, or a mixture of these components, that are all characterised by a high $\mathrm{Z}$ material. What is more, these materials are specific to each particular beam energy. Generally, neither tumours
${ }^{1}$ Laboratory of Nuclear Physics, Faculty of Sciences, Mohammed V University, Rabat, Morocco

*Corresponding author: A. Zeghari Laboratory of Nuclear Physics, Faculty of

Sciences, Mohammed V University, Rabat, Morocco

E-mail: krmzeghari@ gmail.com

Received: 10 April 2018 Accepted: 7 May 2018 
nor patients are flat; therefore, it is necessary to use the FFF beams which can have more benefits than utilizing conventional filtered beams due to the non-uniform intensity across the field size.

A multiple FFF medical linacs (Varian True Beam, Elekta, Siemens...) for energies of 6, $7,10,15$, and $18 \mathrm{MV}$ are basically a standard linacs with the FFF mode. The multiple FFF commercial medical linac was modeled and simulated for the application of stereotactic radiosurgery and radiotherapy, as well as intensity-modulated radiation therapy (IMRT) [1-10].

Recently, many works have been studying the effect of FFF x-rays in the field of physics especially in the clinical domain. For example, using FFF photon beams for treating lung cancer is feasible. Moreover, it decreases beam-on time [11] by $50 \%$ for both intensity-modulated radiation therapy (IMRT) and Rapid Arc modern technique [6-9] that can be an important benefit for the management of organ mobilization. The treatment planning quality using flat conventional x-ray and FFF mode has been reported to be dosimetrically equivalent. The FFF beams are proposed to be applicable to whole-breast irradiation as well.

Many authors have been reviewing the photon energy spectra, backscattering, and beam steering effects of the FFF beams. It has also been reported that other researchers were able to reduce the neutron production in many previous literatures using FFF photon beam. According to these benefits and advantages features, the FFF beams can have a major contribution to decrease unwanted exposure and cause the secondary cancers owing to the absence of the dominant component of flattening filter responsible for scattering radiation in the photon beam [8].

Several studies have found by Monte Carlo simulations that removing the flattening filter from a head linac can cause excessive amounts of contaminating electrons to be excited from the primary collimator and target and passed to the patient surface. To reduce these amounts of contaminating electrons, they include a thin metal plate in the same position as the FF in the head of linac, which is necessary to reduce the electron contaminations.

In our linac model, the thin metal plate is substituted for the flattening filter by a thin plate of aluminium (Al) or tungsten (W) in the beam line that we have obtained a replacement flattening filter (RFF) [1-2, 12]. After entering a plate of $\mathrm{Al}$, a number of electrons have been absorbed and stopped before affecting and entering the patient body [1-2, 13-14].

In this work, the linac's head of Saturne 43 is simulated using BEAMnrc and DOSXYZnrc Monte Carlo (MC) codes. Then, we have studied the effects of RFF mode on the photon energy spectra, percentage depth dose (PDD), beam dose profile, mean energy, and the tissue phantom ratio for the $12 \mathrm{MV}$ unflattened photon beam.

\section{Material and Methods}

\section{Experimental data}

The experimental dosimetric data have been collected in the laboratory NHLB (National Laboratory Henry Becquerel) in France. These dosimetric data include PDD (the dose along the beam axis) and the dose profile (measured at $10 \mathrm{~cm}$ depth of the tank) are measured in a water cubic tank with a dimension of $40 \mathrm{x}$ $40 \times 40 \mathrm{~cm}^{3}$ on a PTW-3100 cylindrical ionization chamber. The entrance surface of the water cubic tank is placed with a distance of $90 \mathrm{~cm}$ from the tungsten target. The generation of a square field must be with a dimension of $10 \times 10 \mathrm{~cm}^{2}$ in $100 \mathrm{~cm}$ from the target. It is recommended that water should be the reference medium for dosimetry in radiotherapy because the biological tissues consist of more than $80 \%$ of water.

Monte Carlo modeling of Saturne 43 linac

The MC modeling and simulation of the 
head's linac is a principal step in radiotherapy field. The MC model constructed and verified can have an advantage to predict a multitude dosimeters characteristics. However, the detailed created and constructed model of a head's linac is a difficult step due to its complex geometry and it is necessary to have a detailed modelling approach to obtain a discrepancy of the dose-calculation of $2 \% / 2 \mathrm{~mm}$ or less in the gamma index method. To fulfill this aim, we used BEAMnrc MC Code to generate and analyze the phase space file registered below the bottom jaws of linac.

In the first step of this work, we simulate precisely the head's geometry of Saturne 43 accordingly to the fabricator's data. In the second step, we modeled the head of Saturne 43 in RFF mode (the complete head's geometry of Saturne 43 without flattening filter) [1-2]. The Linac head components of Saturne 43 are shown in Figure 1. The target was modeled as a slab of W material. The coming electron source hitting the target was modeled as a spot size of $0.07 \mathrm{~cm}$ full-width half-maximum

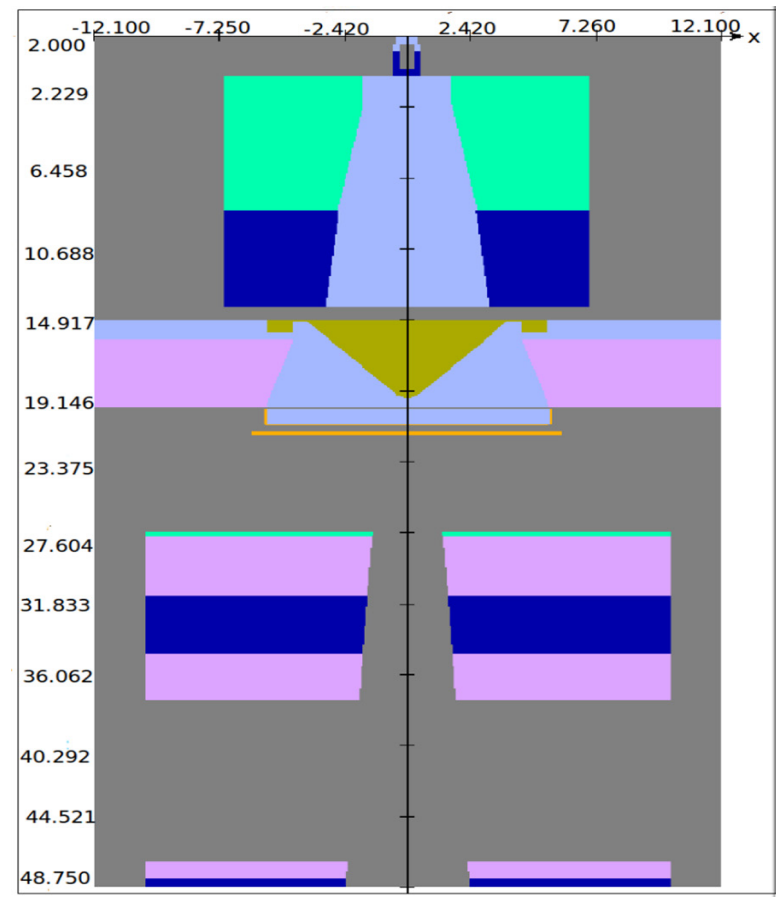

Figure 1: The two-dimensional $X Z$ of the head Saturne 43 modelling by BEAMnrc
(FWHM) with energy of $11.8 \mathrm{MeV}$.

Dose distribution was computed by the DOSXYZnrc MC code on a water phantom using the scored phase space file (PSP) obtained from the BEAMnrc code at $Z=41.5 \mathrm{~cm}$. A cubic water phantom with $(\mathrm{x}, \mathrm{y}, \mathrm{z})$ dimension of $40 \times 40 \times 40 \mathrm{~cm}^{3}$ was then modeled under the treatment head with the DOSXYZnrc user code. The source surface distance (SSD) was $90 \mathrm{~cm}$ between the target component and the phantom surface. The jaws were set to create a field size of $10 \times 10 \mathrm{~cm}^{2}, 5 \times 5 \mathrm{~cm}^{2}$, and 2 $\times 2 \mathrm{~cm}^{2}$ at the distance of $100 \mathrm{~cm}$ from the target component (at the depth of $10 \mathrm{~cm}$ inside the water phantom) [15]. The voxels dimension $(\mathrm{x}, \mathrm{y}, \mathrm{z})$ of the cubic water phantom with the DOSXYZnrc MC code were $5 \times 5 \times 5 \mathrm{~mm}^{3}$ for both depth and profile dose calculations.

The photon and electron low-energy cutoffs parameter used in this simulation were $10 \mathrm{keV}$, and $521 \mathrm{keV}$, respectively.

To investigate the following dosimetric characteristics, including dose profiles at the depth of $10 \mathrm{~cm}$, percentage depth dose (PDD), and photon energy spectra with the different field sizes of $10 \times 10 \mathrm{~cm}^{2}, 5 \times 5 \mathrm{~cm}^{2}$, and $2 \times 2 \mathrm{~cm}^{2}$, the FF component was replaced by a metal plate of $\mathrm{Al}$ or $\mathrm{W}$ of $2 \mathrm{~mm}$ in the modeled linac head on a $12 \mathrm{MV}$ photon beam linac [1-2].

The photon energy spectra on the bottom jaws were calculated using the scored PSF obtained from the BEAMnrc code at $\mathrm{Z}=50 \mathrm{~cm}$ with BEAMDP( BEAM Data Processor) user code for FF and RFF modes using 100 energy bins.

The photon energy spectra from PSF was obtained using the BEAMDP code and graphs were plotted with the $2 \mathrm{D}$ graph plotting software QT-GRACE.

The initial histories were $3 \times 10^{9}$ particles on a $10 \times 10 \mathrm{~cm}^{2}$ field size. Simulations using the BEAMnrc and DOSXYZnrc MC code were run on a desktop core i7 CPU with $8 \mathrm{GHz}$ RAM on Ubuntu 14.04 system. The time was 144 hours for every PSF obtained by FF mode and 8 hours for every PSF with RFF mode. 


\section{Results}

The statistical uncertainty of the MC BEAMnrc and DOSXYZnrc codes results was less than $1 \%$ for the depths from $\mathrm{z}=0 \mathrm{~cm}$ to $\mathrm{z}=25$ $\mathrm{cm}$. The discrepancy between measurements and MC for PDD and DP data was $1.5 \% / 1 \mathrm{~mm}$ in the gamma index method in our previous published work (A. Zeghari, R. Saaidi, and R. Cherkaoui El Moursly, Modeling of a Linear Accelerator Saturne 43 and Study of Photon Dose Distributions, 4th International Conference on Automation, Control Engineering and Computer Science (ACECS - 2017) Proceedings of Engineering and Technology).

Figure 2 shows a comparison of $\mathrm{MC}$ which calculated PDD for FF and RFF modes and experimental results versus water phantom for $12 \mathrm{MV}$ photon beam of Saturne 43 linac with $10 \times 10 \mathrm{~cm}^{2}$ field size. At the depth of $\mathrm{z}=$ $0.5 \mathrm{~cm}$, the fractional buildup dose was 31.56 $\%$ while it was $39.92 \%$ for RFF mode. This demonstrated that for the $12 \mathrm{MV}$ with RFF mode, the dose was higher than the flattened beam at depth of $0.5 \mathrm{~cm}$.

The profile dose data in the Figure 3 was normalized to the dose at central axis - depth of $10 \mathrm{~cm}$ [15]. Beam profile data on $10 \times 10 \mathrm{~cm}^{2}$ square field size were calculated at a depth of

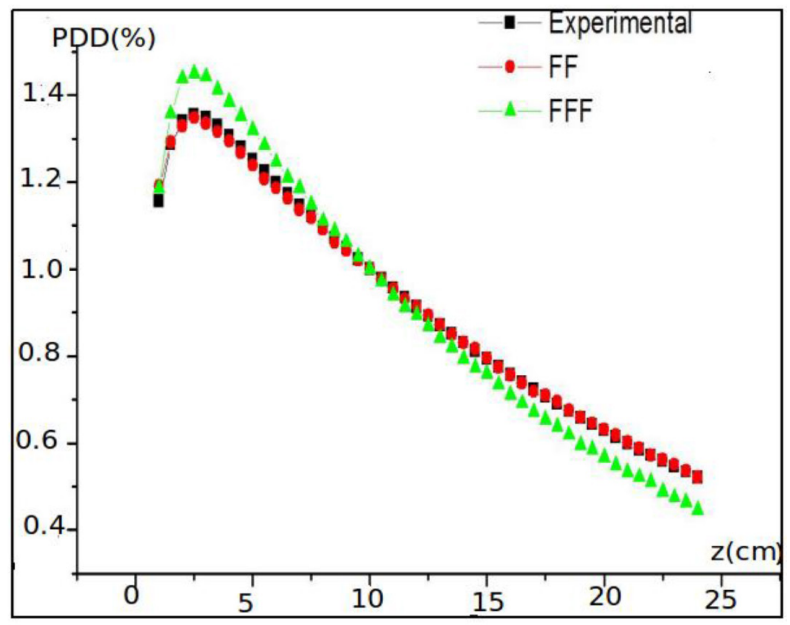

Figure 2: comparison between PDD curve for experiment, FF, and FFF modes results in the water phantom for a $12 \mathrm{MV}$ photon beam with a square field size of $10 \times 10 \mathrm{~cm}^{2}$.

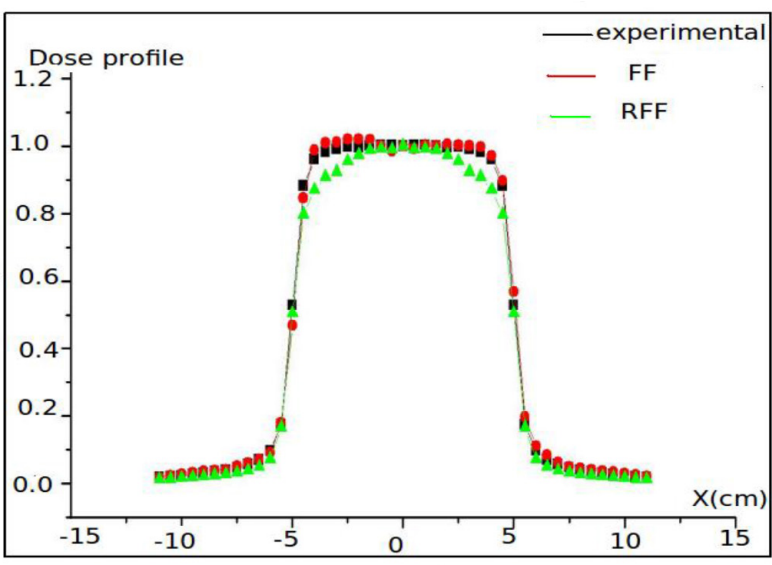

Figure 3: Comparison between cross-plane profiles dose for experiment, FF, and RFF modes results in the water phantom at 10 $\mathrm{cm}$ depth with the $10 \times 10 \mathrm{~cm}^{2}$ field size for $12 \mathrm{MV}$ photon beam.

$10 \mathrm{~cm}$ for both FF and RFF modes.

The Figure 3 illustrates the dose profile of experimental data for both FF and RFF modes at a depth of $10 \mathrm{~cm}$ on $10 \times 10 \mathrm{~cm}^{2}$ square field size for $12 \mathrm{MV}$ photon beam energy.

Figures 4, 5 and 6 show the photon energy spectra for FF and RFF modes on the bottom jaws of Saturne 43 linac head for field sizes of $10 \times 10 \mathrm{~cm}^{2}, 5 \times 5: \mathrm{cm}^{2}$, and $2 \times 2 \mathrm{~cm}^{2}$, respectively.

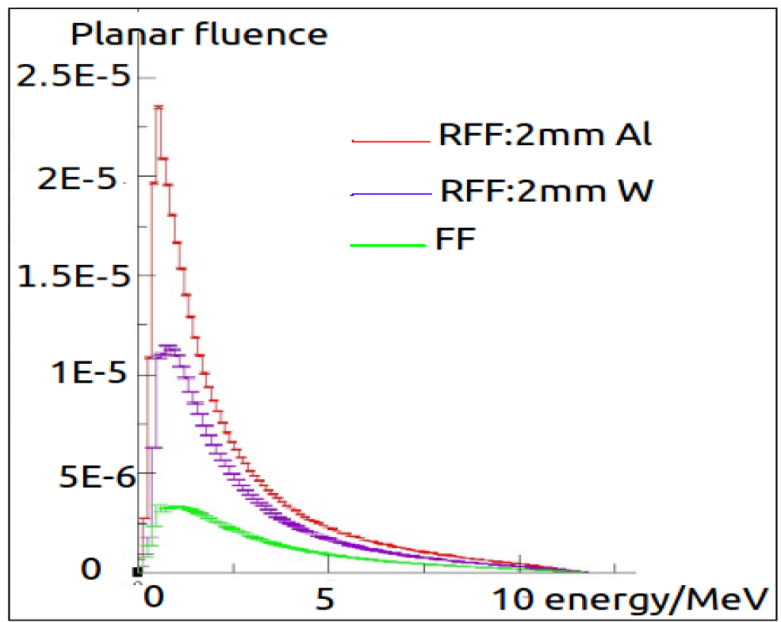

Figure 4: photon fluence spectra: $2 \mathrm{~mm}$ of Al RFF mode, $2 \mathrm{~mm}$ of $\mathrm{W}$ RFF mode, and FF beam with the field size $10 \times 10 \mathrm{~cm}^{2}$ for 12 MV photon beam. 


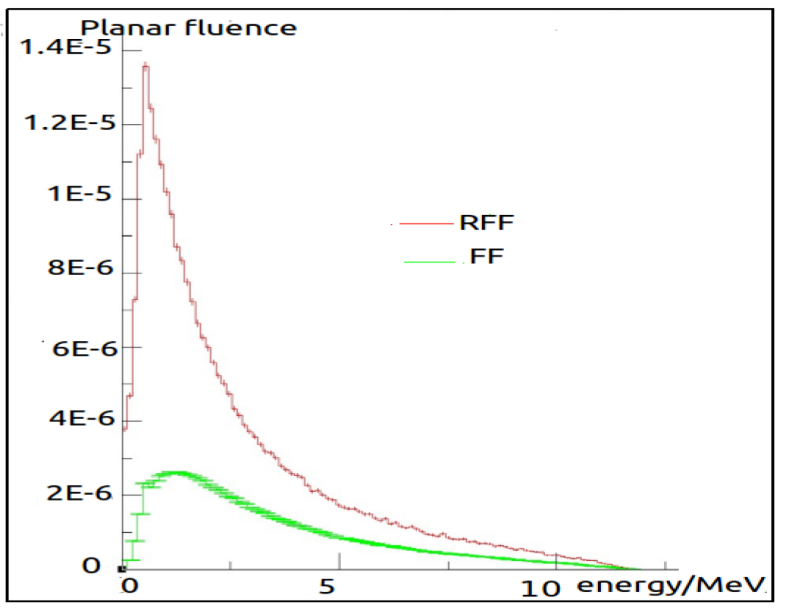

Figure 5: photon fluence spectra: RFF mode and FF mode with the field size $5 \times 5 \mathrm{~cm}^{2}$ for $12 \mathrm{MV}$ photon beam.

Figure 7 shows the electron energy spectra for FF and RFF modes on the bottom jaws of Saturne 43 linac head for field size of $10 \times 10$ $\mathrm{cm}^{2}$.

The photon fluence spectra were obtained by the BEAMDP user code analyzing the phase space file below the lower jaws for the following field sizes $10 \times 10 \mathrm{~cm}^{2}, 5 \times 5 \mathrm{~cm}^{2}$, and $2 \times$ $2 \mathrm{~cm}^{2}$, respectively.

The beam quality index (Q) specified by $\mathrm{TPR}_{20 / 10}[12,14-15]$ for FF mode was kept the

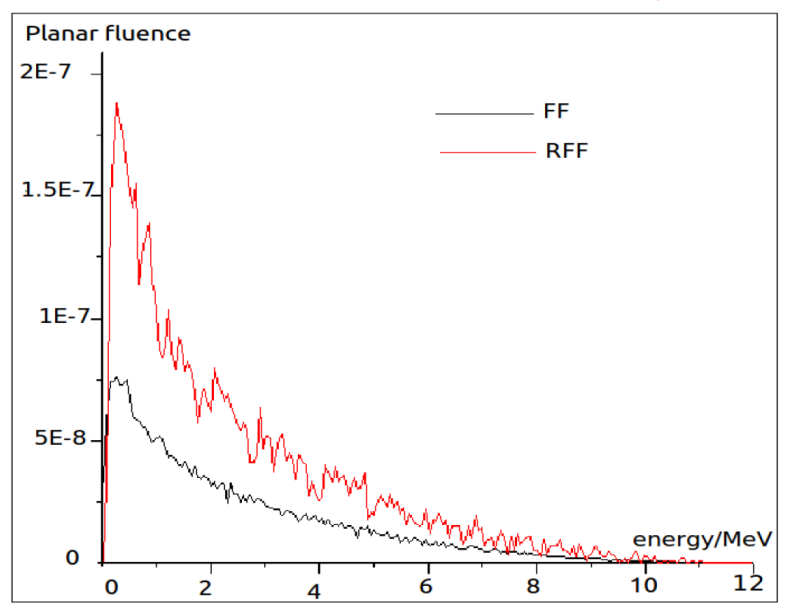

Figure 7: Electron fluence spectra for RFF mode and FF mode with the field size $10 \times$ $10 \mathrm{~cm}^{2}$ for $12 \mathrm{MV}$ photon beam.

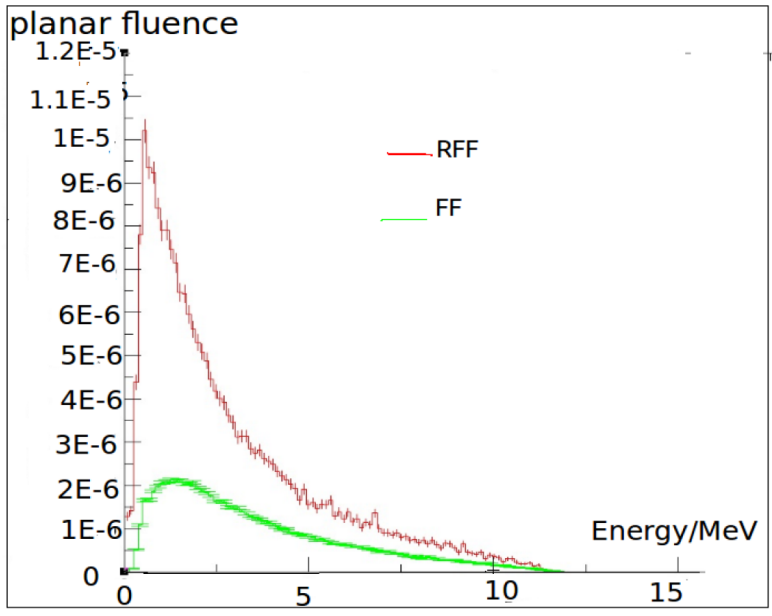

Figure 6: photon fluence spectra: RFF mode and FF mode with the field size $2 \times 2 \mathrm{~cm}^{2}$ for $12 \mathrm{MV}$ photon beam.

same for RFF mode defined as the absorbed dose ratio to water at the depth of 10 and 20 $\mathrm{cm}$ inside a water phantom along the beam axis. $\mathrm{TPR}_{20 / 10}$ calculates the effective attenuation coefficient of the photon fraction inside the water phantom, and approximately indicates the description exponential decrease of the photon depth-dose curve and it is defined by the following equation:

$$
\mathrm{TPR}_{20,10}=1.2661 * \mathrm{PDD}_{20,10}-0.0595
$$

$\mathrm{TPR}_{20,10}$ was calculated for FF and RFF modes for Al metal plate of $2 \mathrm{~mm}$ or by a W metal plate of $2 \mathrm{~mm}$, which were to be 0.73 , 0.66 and 0.67 , respectively.

The calculated mean energies for all photon energy spectra for the RFF case were 2.48 $\mathrm{MeV}$ for Al metal plate of $2 \mathrm{~mm}$ and $2.74 \mathrm{MeV}$ for a W metal plate of $2 \mathrm{~mm}$ on $10 \times 10 \mathrm{~cm}^{2}$ field size.

\section{Discussion}

The Figure 2 shows the PDD data obtained on $10 \times 10 \mathrm{~cm}^{2}$ square field size. In this figure, a difference between PDD values is observed that increased along the $\mathrm{z}$ axis. This results demonstrate that the PDD values for the RFF mode are slightly higher than FF mode on the same voxels along the $\mathrm{z}$ axis. The electron 
contaminations have a significant effect in the build-up region and phantom surface and also lead to higher fluence of contamination electrons with RFF mode.

The dose rates at the maximum depth between filtered and unfiltered beams were calculated as the ratio of absorbed dose between the FF and RFF modes at the depth of maximum dose $z_{\text {Dmax }}$ with the same number of initial electrons incident. The unfiltered dose rates were 3.94 and 2.74 times higher for 2 $\mathrm{mm}$ metal plate of $\mathrm{Al}$ and $2 \mathrm{~mm}$ for $\mathrm{W}$ metal plate, respectively on a $10 \times 10 \mathrm{~cm}^{2}$ field size. Other authors found that for RFF mode, the dose rate increased by 5.5 and 2.31 for 18 [16] and $6 \mathrm{MV}$, respectively. Moreover, for a linac of $15 \mathrm{MV}$, the percentage depth dose rate increased by a factor of about 4 with field size of $10 \times 10 \mathrm{~cm}^{2}[3-7,9,11,12,17]$. A study of a linac of $10 \mathrm{MV}$ beam $[14,18]$ at the depth of $\mathrm{Z}_{\mathrm{Dmax}}$ showed that the difference varied from $3.4 \%$ to $10.7 \%$ for the $25 \times 25 \mathrm{~cm}^{2}$ and $2 \times$ $2 \mathrm{~cm}^{2}$ fields, respectively. For a $6 \mathrm{MV}$ beam [14], the dose increased from $6.0 \%$ to $14.6 \%$ for $25 \times 25 \mathrm{~cm}^{2}$ and $2 \times 2 \mathrm{~cm}^{2}$ square field sizes. The beam energy difference between the FFF and FF modes is the essential cause for the superficial dose difference between the FF and FFF modes, that FFF produces a higher superficial dose.

In another study, the buildup (defined between the depth of $\mathrm{z}=0 \mathrm{~cm}$ and $\mathrm{z}=1.5 \mathrm{~cm}$ ) dose for energy $6 \mathrm{MV}$ has showed a slight expanded dose on the both of square field sizes $2 \times 2 \mathrm{~cm}^{2}$ and $10 \times 10 \mathrm{~cm}^{2}$ for FFF mode than that with FF mode. However, the difference was not important. In the buildup region, at the depth of $\mathrm{z}=0.5 \mathrm{~cm}$, the fractional buildup doses for 6 MV FF mode varied from $85 \%$ to $87 \%$ which did not have an important distinction in comparison with $6 \mathrm{MV}$ FFF mode which varied from $90 \%$ to $91 \%$. A study for energy $10 \mathrm{MV}$ also has leaded to a slight augmentation with FFF mode than that with FF mode for different depths in the buildup region. In the depth of $\mathrm{z}=0.2 \mathrm{~cm}$, for $10 \mathrm{MV}$ flattened photon beam on a field size $10 \times 10 \mathrm{~cm}^{2}$, the dose increased from $20 \%$ to $49 \%$. In parallel, for $10 \mathrm{MV} \mathrm{FFF}$ photon beam on the same field size, the fractional dose increased from $24 \%$ to $57 \%$. At the depth of $\mathrm{z}=0.5 \mathrm{~cm}$ for field size $10 \times 10 \mathrm{~cm}^{2}$, the fractional doses in the buildup region for both $10 \mathrm{MV} \mathrm{FF}$ and $10 \mathrm{MV} \mathrm{FFF}$ modes varied from $75 \%$ to $78 \%$. At the same depth for field size $2 \times 2 \mathrm{~cm}^{2}$, the fractional doses varied between $67 \%$ and $72 \%$. The advantages of the FFF mode consist of higher dose rate in surface and buildup regions and the decrease in the leakage of radiation photon in expanded treatment field. The using of FFF mode can be an advantage of treatment some types of cancer as prostate, brain, larynx, and pharynx, cranial or extra-cranial stereotactic treatments with small and medium field sizes. In the cubic water phantom, the dose can be precisely calculated at any depths in the buildup and surface region. In contrary with clinical cases using the CT dataset, it is not easy to precisely measure the surface and buildup dose of the patient's dose.

Dose profile distribution was computed at depth of $10 \mathrm{~cm}$ on a $10 \times 10 \mathrm{~cm}^{2}$ square field size. The dose profile distribution in the penumbra region between the RFF and FF modes was slightly different, but the difference is not important $[4,10,11,14]$.

The distribution of the photon fluence and relative dose with RFF mode would be lower than dose received by the non-targeted area.

The distribution of the profile dose in the out-of-field region was computed. It found that the profile dose distribution in this region was lower for RFF mode (Figure 3). This reason was supported in other studies by many authors [3-11, 13, 15, 16, 19].

$\mathrm{TPR}_{20,10}$ data calculated for $\mathrm{FF}$ and RFF modes for an Al metal plate of $2 \mathrm{~mm}$ or by a W metal plate of $2 \mathrm{~mm}$ were $0.73,0.66$, and 0.67 , respectively. It is seen that $\mathrm{TPR}_{20,10}$ slightly decreased [4, 13-19] for RFF beams. Some authors exploited the relationship between beam quality specifies and stopping- 
power-ratios of FFF beams and demonstrated that using $\% \operatorname{dd}(10)_{x}$ as beam quality specifier Spencer Attix stops power ratios for both FFF and FF modes leading to the same values of $\% \operatorname{dd}(10)_{\mathrm{x}}$ within the acceptable calculation errors [14].

The ratios of photon fluence of RFF / FF beams were $7.44,5.22$ and 4.74 for the field size of $10 \times 10 \mathrm{~cm}^{2}, 5 \times 5 \mathrm{~cm}^{2}$ and $2 \times 2 \mathrm{~cm}^{2}$, respectively. This results for the ratios of photon fluence demonstrated that the photon fluence increases with the field size [9, 16, 18]. The flattening filter component has an important responsibility of attenuation of photon fluence with FF mode. An important fraction of photons with low energy was absorbed by the thick central part of the flattening filter component, but with decreasing field size. Thus less fraction of photons with low energy can pass of thin lateral part of flattening filter component.

Our study shows that energy spectra with the FF mode are less soft than RFF mode. The calculated mean energy for all spectra shows that mean energy for the RFF case decreases from 3.29 to $2.48 \mathrm{MeV}$ for Al metal plate for $2 \mathrm{~mm}$ and 3.29 to $2.74 \mathrm{MeV}$ for a $\mathrm{W}$ metal plate of $2 \mathrm{~mm}$ on a $10 \times 10 \mathrm{~cm}^{2}$ field size. The bremsstrahlung photons with low-energy level produced inside the target were absorbed by the two components, including flattening filter and primary collimator. When the flattening filter was replaced by a metal plate of $\mathrm{Al}$, low-energy photons and the contaminating electrons can pass into the patient body and then contribute to the treatment beam. The contaminating electrons increased for RFF mode by $246.4 \%$ in comparison to FF mode on a field size of $10 \times 10 \mathrm{~cm}^{2}$.

The mean energy of the flattened beam decreased by a ratio of 1.33 for a metal plate of $\mathrm{Al}$ and 1.2 for a metal plate of $\mathrm{W}$ with $10 \times 10$ $\mathrm{cm}^{2}$ field size. We conclude that the mean energy decreased for the RFF mode. Many studies have been carried out on Elekta precise and Varian clinac 2100C linacs. There were some differences between previous works for mean energy and photon energy spectra with FFF mode $[1,2,8,14,16-18,20]$. The most previous studies were based on simulation with FF removed from conventional flattened beam accelerators as our work but not on a real commercially clinical accelerators with FFF capability.

\section{Conclusion}

We have developed two models with FF and RFF modes for head's linac Saturne 43 with energy of $12 \mathrm{MV}$ photon mode using the BEAMnrc MC User code. Our PDD calculations at the depth of maximum dose for RFF mode found an increase factor more than 3.94 in the dose rate with $10 \times 10 \mathrm{~cm}^{2}$ field size. This increase in the buildup region dose for RFF mode was owing to contamination electrons generated in the RFF mode. Our computation of the dose profiles distribution has revealed a decrease in out of field doses for unflattened beams. The time was reduced more than $50 \%$ for the RFF mode. The spectra of photon energy for flattened and unflattened modes were significantly different for all field sizes. In addition, the mean energy and the tissue phantom ratio decreased, and the photon fluence for the RFF mode was higher than FF mode. In conclusion, the actual paper demonstrates directions for future researches related to the original questions about dosimetric properties of FFF beam of Saturne 43 linac head.

\section{Acknowledgment}

The authors would like to thank Mr. LE ROY Maïwenn for providing the data from LNHB-CEA.

\section{Conflict of Interest}

None

\section{References}

1. Bencheikh M, Maghnouj A, Tajmouati J. Energetic properties' investigation of removing flattening filter at phantom surface: Monte Carlo study using BEAMnrc code, DOSXYZnrc code and BEAMDP code. 
Physics of Particles and Nuclei Letters. 2017;14:95362. doi: 10.1134/s1547477117060073.

2. Bencheikh M, Maghnouj A, Tajmouati J. Photon beam softening coefficients evaluation for a $6 \mathrm{MeV}$ photon beam for an aluminum slab: Monte Carlo study using BEAMnrc Code, DOSXYZnrc Code, and BEAMDP code. Moscow University Physics Bulletin. 2017;72:263-70. doi: 10.3103/s0027134917030043.

3. Cashmore J, Golubev S, Dumont JL, Sikora M, Alber M, Ramtohul M. Validation of a virtual source model for Monte Carlo dose calculations of a flattening filter free linac. Med Phys. 2012;39:3262-9. doi: 10.1118/1.4709601. PubMed PMID: 22755709.

4. Hrbacek J, Lang S, Klock S. Commissioning of photon beams of a flattening filter-free linear accelerator and the accuracy of beam modeling using an anisotropic analytical algorithm. Int $J$ Radiat Oncol Biol Phys. 2011;80:1228-37. doi: 10.1016/j. ijrobp.2010.09.050. PubMed PMID: 21129855.

5. Kry SF, Vassiliev ON, Mohan R. Out-of-field photon dose following removal of the flattening filter from a medical accelerator. Phys Med Biol. 2010;55:215566. doi: 10.1088/0031-9155/55/8/003. PubMed PMID: 20305334.

6. Xiao Y, Kry SF, Popple R, Yorke E, Papanikolaou N, Stathakis $S$, et al. Flattening filter-free accelerators: a report from the AAPM Therapy Emerging Technology Assessment Work Group. J Appl Clin Med Phys. 2015;16:5219. doi: 10.1120/jacmp.v16i3.5219. PubMed PMID: 26103482; PubMed Central PMCID: PMC5690108.

7. Tsiamas P, Seco J, Han Z, Bhagwat M, Maddox J, Kappas $C$, et al. A modification of flattening filter free linac for IMRT. Med Phys. 2011;38:2342-52. doi: 10.1118/1.3571419. PubMed PMID: 21776768.

8. Zhang DG, Feygelman V, Moros EG, Latifi K, Hoffe $S$, Frakes J, et al. Superficial and peripheral dose in compensator-based FFF beam IMRT. J App/ Clin Med Phys. 2017:18:151-6. doi: 10.1002/acm2.12018. PubMed PMID: 28291940; PubMed Central PMCID: PMC5689887.

9. Zwahlen DR, Lang S, Hrbacek J, Glanzmann C, Kloeck S, Najafi Y, et al. The use of photon beams of a flattening filter-free linear accelerator for hypofractionated volumetric modulated arc therapy in localized prostate cancer. Int J Radiat Oncol Biol Phys. 2012;83:1655-60. doi: 10.1016/j.jijobp.2011.10.019. PubMed PMID: 22572080.

10. Sidek J, Ariff M. Monte Carlo investigations of radiotherapy beams: studies of conventional, stereotactic and unflattened beams. Birmingham: University of Birmingham; 2010.

11. Georg D, Knoos T, McClean B. Current status and future perspective of flattening filter free photon beams. Med Phys. 2011;38:1280-93. doi: 10.1118/1.3554643. PubMed PMID: 21520840.

12. Fogliata A, Garcia R, Knoos T, Nicolini G, Clivio A, Vanetti $E$, et al. Definition of parameters for quality assurance of flattening filter free (FFF) photon beams in radiation therapy. Med Phys. 2012;39:6455-64. doi: 10.1118/1.4754799. PubMed PMID: 23039680.

13. Kuess P, Georg D, Palmans H, Lechner W. Technical Note: On the impact of the incident electron beam energy on the primary dose component of flattening filter free photon beams. Med Phys. 2016;43:4507. doi: 10.1118/1.4954849. PubMed PMID: 27487867.

14. Budgell G, Brown K, Cashmore J, Duane S, Frame J, Hardy M, et al. IPEM topical report 1: guidance on implementing flattening filter free (FFF) radiotherapy. Phys Med Biol. 2016;61:8360-94. doi: 10.1088/0031-9155/61/23/8360. PubMed PMID: 27819253.

15. Radiology IAEAWHOPAHOESOT, ESTRO. OIWP. Absorbed Dose Determination in External Beam Radiotherapy: An International Code of Practice for Dosimetry Based on Standard of Absorbed Dose to Water. Vienna: International Atomic Energy Agency; 2000.

16. Mesbahi A, Nejad FS. Monte Carlo study on a flattening filter-free 18-MV photon beam of a medical linear accelerator. Radiat Med. 2008;26:331-6. doi: 10.1007/s11604-008-0234-y. PubMed PMID: 18677606.

17. Mesbahi A. Dosimetric characteristics of unflattened $6 \mathrm{MV}$ photon beams of a clinical linear accelerator: a Monte Carlo study. Appl Radiat Isot. 2007;65:102936.doi: 10.1016/j.apradiso.2007.04.023.

18. Javedan K, Feygelman V, Zhang RR, Moros EG, Correa CR, Trotti A, et al. Monte Carlo comparison of superficial dose between flattening filter free and flattened beams. Phys Med. 2014;30:503-8. doi: 10.1016/j.ejmp.2014.03.001. PubMed PMID: 24662096.

19. Shende R, Gupta G, Patel G, Kumar S. Commissioning of TrueBeamTM medical linear accelerator: quantitative and qualitative dosimetric analysis and comparison of flattening filter (FF) and flattening filter free (FFF) Beam. International Journal of Medical Physics, Clinical Engineering and Radiation Oncology. 2016:5:51-69.doi: 10.4236/ijmpcero.2016.51006.

20. Meshram MN, Pramanik S, Ranjith CP, Gopal SK, Dobhal R. Dosimetric properties of equivalent-quality flattening filter-free (FFF) and flattened photon beams of Versa HD linear accelerator. J Appl Clin Med Phys. 2016;17:358-70. doi: 10.1120/jacmp.v17i3.6173. PubMed PMID: 27167293; PubMed Central PMCID: PMC5690903. 